\title{
Confluent and reticulated papillomatosis manifested as vertically rippled and keratotic plaques
}

\author{
Zhong-Sheng Min ${ }^{1}$, Cheng Tan ${ }^{1}$, Ping Xu1, Wen-Yuan Zhu ${ }^{2}$

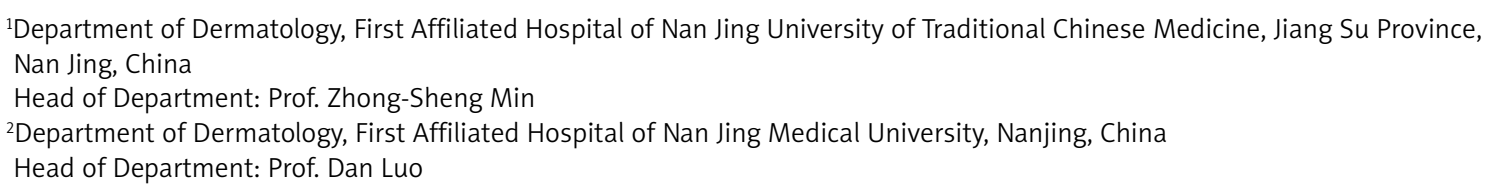

Postep Derm Alergol 2014; XXXI, 5: 335-337

DOI: 10.5114/pdia.2014.44011

Confluent and reticulated papillomatosis (CRP) is a rare dermatosis of uncertain etiology that preferentially affects young adults. Eruptions usually present as grayish brown, flat elevated, keratotic papules and plaques that are confluent centrally and reticulated peripherally. It most commonly involves the intermammary and epigastric regions. We reported a young boy of CRP manifesting vertically rippled and keratotic streaks on his trunk.

An 18-year-old boy was admitted to our dermatology department with vertically rippled and keratotic eruptions on the trunk. Two years ago, the patient noticed light brown and rippled streaks on his epigastric area. It became abruptly spreading on the chest, the back and waist during recent 6 months. There were no itching or other complaints. His past medical history was noncontributory. At the time of our observation, his height and weight were $170 \mathrm{~cm}$ and $89 \mathrm{~kg}$, respectively, with a body mass index equal to $30.8 \mathrm{~kg} / \mathrm{m}^{2}$ (normal value $<25 \mathrm{~kg} / \mathrm{m}^{2}$ ). Cutaneous examination showed brownish-black, vertically rippled and keratotic streaks involving his abdomen and the waist. In addition, red papules and plaques were seen around the axilla and antecubital fossae (Figures 1 and 2). An unstained specimen and culture of fine scale obtained from skin scrapings was negative. No fluorescence was observed in the affected skin under a Wood light examination. The biopsy specimens taken from the epigastric region revealed slight basket-weave hyperkeratosis, squat papillomatosis and a slight perivascular mononuclear infiltration in the upper dermis (Figure 3). Amyloid deposition was not identified with Congo red stain. The results of the laboratory evaluation and chemistry profile were within normal limits, and no endocrine imbalance including diabetes mellitus was detected.
The diagnosis of CRP was finally made on the basis of supportive histology and characteristic clinical features except its rippled appearance. We initiated treatment with Calcipotriol cream twice daily for 2 months. There was no improvement, and treatment was switched to isotretinoin with an initial dose of $30 \mathrm{mg}$ on alternate days. In the $3^{\text {rd }}$ month of follow-up, partial flattening and fading of the lesions was observed. Complete resolution was still not obtained at the time of the $5^{\text {th }}$ month and the patient was unwilling to accept any further treatment.

Confluent and reticulated papillomatosis is an uncommon skin disorder initially described by Gougerot and Carteaud in 1932 [1]. The disease begins in the late teens or early twenties, and has been reported worldwide and in all racial groups.

The lesions typically begin as round, flat-topped, sharply defined red or brown papules that can become hyperkeratotic and verrucous. It spreads centrifugally, leading to confluent lesions in the center and reticulated ones at the periphery. It can also manifest as atrophic macules with a shiny quality or with surface change resembling cigarette paper. The most common sites of involvement are the inframammary area, breasts, axillae, neck, abdomen, interscapular area and inguinal region. Rarely, CRP develops at the other sites, such as the knee, elbow, hand, and antecubital and popliteal fossae. Eruptions in CRP are usually asymptomatic, but mild pruritic ones had also been described. Rarely CRP can manifest as rippled reticulated erythema in Caucasians [2, 3]. In our case, the eruption initially consisted of confluent, flat, brown papules localized primarily in the epigastric area with a subsequent spread to the waist. Most lesions were vertically rippled. To the best of my knowledge, this is the second CRP case manifesting as rippled streaks

Address for correspondence: Cheng Tan MD, Department of Dermatology, First Affiliated Hospital of Nan Jing University of Traditional Chinese Medicine, 155 Hanzhong Road, 210029 Nanjing, China, phone: +86 13515102233, e-mail: tancheng@medmail.com.cn Received: 22.01.2014, accepted: 26.02.2014. 


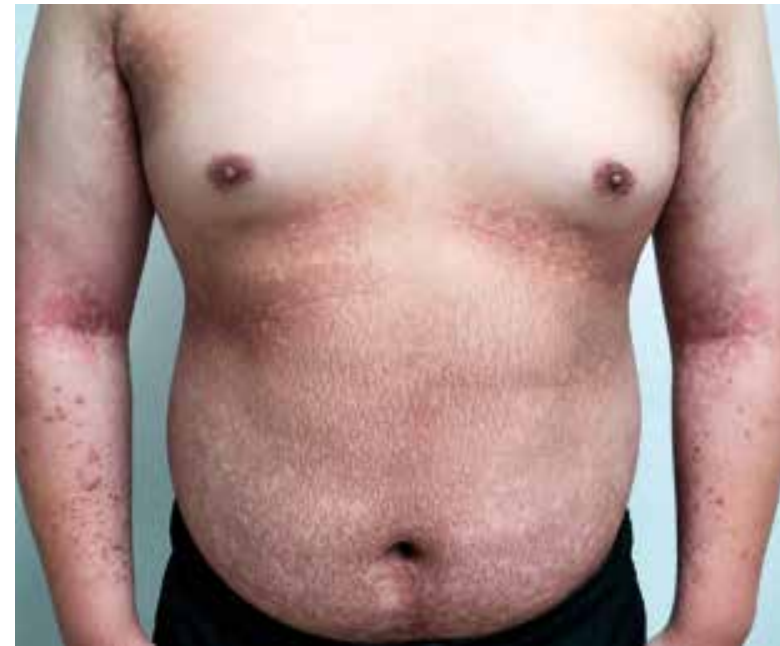

Figure 1. Most of the lesions were present on the abdomen and the waist

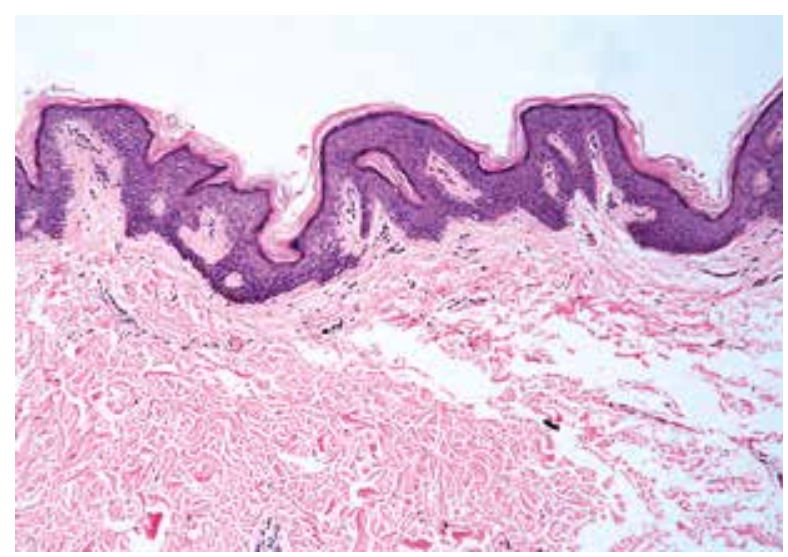

Figure 3. Slight basket-weave hyperkeratosis, squat papillomatosis and a slight perivascular mononuclear infiltration in the upper dermis $(H+E 100 x)$

and the first non-Caucasian one as well. Skin biopsy in CRP showed hyperkeratosis, slight papillomatosis with acanthosis, with few inflammatory cells infiltration in the dermis. A acid-Schiff stain is predicted to be negative [1, 3]. Electron microscopic study revealed that increased melanin pigmentation in CRP is resulted from increased melanosomes present in the hyperkeratotic horny layer [4]. Our patient met these criteria with the exception of its rippling appearance.

The differential diagnosis for our patient was wide and included acanthosis nigricans, Prurigo pigmentosa, Darier's disease, tinea versicolor and amyloidosis etc. [1, 5]. Acanthosis nigricans was not favored due to lack of histologic evidence of papillomatosis resulting from the upward projection of finger-like dermal papillae which are covered by thinned epidermis. Prurigo pigmentosa can be excluded due to the lack of histological evidence

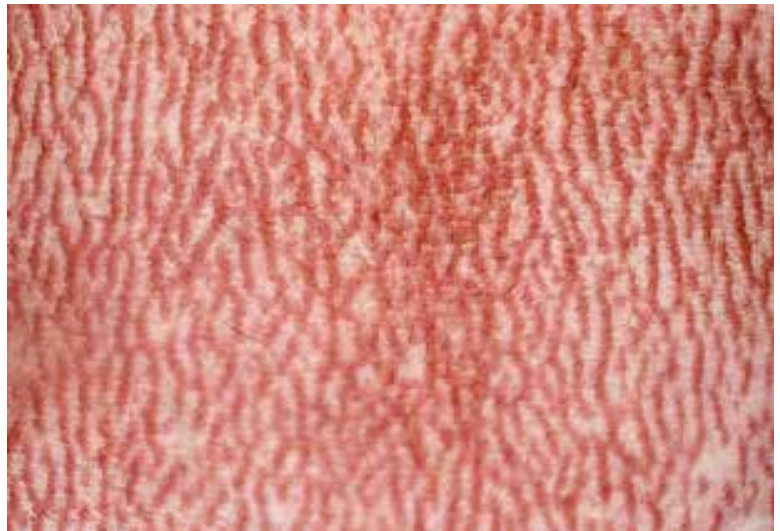

Figure 2. The lesions were brownish-black keratotic streaks that rippled vertically

for vacuolar alteration, dyskeratotic cells or a lichenoid infiltrate of lymphocytes. Darier's disease was ruled out due to the lack of suprabasal acantholysis and dyskeratotic cells. Tinea versicolor was unlikely considering its absence of organisms on $\mathrm{KOH}$ examination and Periodic acid-Schiff (PAS) staining. Macular amyloidosis was not possible without pathological signs of amyloid deposits.

The etiology and pathogenesis of CRP are not precisely known. Several theories for the cause of CRP have been suggested, including hereditary predisposition, exposure to ultraviolet, endocrine imbalances, disturbance of keratinization and infection of the skin with Pityrosporum ovale or other fungal [2]. As far our patient is concerned, no triggering factor had been hitherto identified.

Confluent and reticulated papillomatosis can be recalcitrant to present therapies, including salicylic acid, topical or systemic steroids, vitamin $D_{3}$ analogues, vitamin A derivatives, selenium sulfide, liquid nitrogen, progesterone, coal tar, dermabrasion, hydroquinone, phototherapy, 5-fluorouracil, thyroid extracts, anti-fungal agents, and antibiotics such as minocycline. However, we observed a marked improvement with the administration of isotretinoin for a period of 5 months.

Confluent and reticulated papillomatosis might present with rippled keratotic streaks, and every dermatologist should be aware of such rare demonstrations.

\section{Acknowledgments}

This work was supported by the National Natural Science Foundation of China (Grant No. 81173400)

\section{References}

1. Scheinfeld N. Confluent and reticulated papillomatosis: a review of the literature. Am J Clin Dermatol 2006; 7: 305-13.

2. Tamraz H, Raffoul M, Kurban M, et al. Confluent and reticulated papillomatosis: clinical and histopathological study of 10 cases from Lebanon. J Eur Acad Dermatol Venereol 2013; 27: e119-23. 
3. Erkek E, Ayva S, Atasoy P, Emeksiz MC. Confluent and reticulated papillomatosis: favourable response to low-dose isotretinoin. J Eur Acad Dermatol Venereol 2009; 23: 1342-3.

4. Jimbow M, Talpash O, Jimbow K. Confluent and reticulated papillomatosis: clinical, light and electron microscopic studies. Int J Dermatol 1992; 31: 480-3.

5. Mutasim DF. Confluent and reticulated papillomatosis without papillomatosis. J Am Acad Dermatol 2003; 49: 1182-4. 